\title{
A Generalized Strong Convergence Algorithm in the Presence of Errors for Variational Inequality Problems in Hilbert Spaces
}

\author{
Mostafa Ghadampour $\mathbb{D}^{1},{ }^{1}$ Donal O’Regan $\mathbb{D}^{2},{ }^{2}$ Ebrahim Soori $\mathbb{D}^{1},{ }^{1}$ and Ravi P. Agarwal $\mathbb{D}^{3}$ \\ ${ }^{1}$ Department of Mathematics, Lorestan University, Lorestan, Khoramabad, Iran \\ ${ }^{2}$ School of Mathematics, Statistics, National University of Ireland, Galway, Ireland \\ ${ }^{3}$ Department of Mathematics Texas A\&M University-Kingsville, 700 University Blvd., MSC 172 Kingsville, Texas, USA
}

Correspondence should be addressed to Ebrahim Soori; sori.e@lu.ac.ir

Received 3 April 2021; Revised 8 May 2021; Accepted 9 June 2021; Published 21 June 2021

Academic Editor: Ismat Beg

Copyright (c) 2021 Mostafa Ghadampour et al. This is an open access article distributed under the Creative Commons Attribution License, which permits unrestricted use, distribution, and reproduction in any medium, provided the original work is properly cited.

In this paper, we study the strong convergence of an algorithm to solve the variational inequality problem which extends a recent paper (Thong et al., Numerical Algorithms. 78, 1045-1060 (2018)). We reduce and refine some of their algorithm conditions and we prove the convergence of the algorithm in the presence of some computational errors. Then, using the MATLAB software, the result will be illustrated with some numerical examples. Also, we compare our algorithm with some other well-known algorithms.

\section{Introduction}

Let $H$ be a real Hilbert space with the inner product $\langle.$, . $\rangle$ and the norm $\|$.$\| , and C$ be a nonempty, closed, and convex subset of $H$. The variational inequality (VI) is to find a point $x \in C$ such that

$$
\langle A x, y-x\rangle \geq 0, \forall y \in C
$$

where $A$ is a mapping of $\mathrm{C}$ into $H$. The solution set of (1) is denoted by $\operatorname{VI}(A, C)$. Variational inequalies arise in the study of network equilibriums, optimization problems, saddle point problem, Nash equilibrium problems in noncooperative games etc.; see, for example, [1-12] and the references therein.

A new algorithm was proposed by Korpelevich [13] for solving the problem (VI) in the Euclidean space which is known as the extragradient method. Let $x_{1}$ be an arbitrary element in $H$ and consider

$$
\left\{\begin{array}{l}
y_{n}=P_{C}\left(x_{n}-\lambda A x_{n}\right) \\
x_{n+1}=P_{C}\left(x_{n}-\lambda A y_{n}\right)
\end{array}\right.
$$

where $\lambda$ is a number in $(0,1), P_{C}$ is the Euclidean least distance projection of $H$ onto $C$, and $A: C \longrightarrow H$ is a monotone operator. The next algorithm (3) was introduced by Tseng [14] and applying the modified forward-backward (F-B) method is a good alternative to the extragradient method (TEGM):

$$
\left\{\begin{array}{l}
y_{n}=P_{C}\left(x_{n}-\lambda A x_{n}\right), \\
x_{n+1}=P_{X}\left(y_{n}-\lambda\left(A y_{n}-A x_{n}\right)\right),
\end{array}\right.
$$

where $X=C$ and $X=H$ if $A$ is Lipschitz continuous. The following algorithm (4) was proposed by Shehu and Iyiola [15] which a viscosity type subgradient extragradient method (VSEGM):

$$
\left\{\begin{array}{l}
y_{n}=P_{C}\left(x_{n}-\lambda_{n} A x_{n}\right), \\
T_{n}=\left\{z \in H:\left\langle x_{n}-\lambda_{n} A x_{n}-y_{n}, z-y_{n}\right\rangle \leq 0\right\}, \\
z_{n}=P_{T_{n}}\left(x_{n}-\lambda_{n} A y_{n}\right), \\
x_{n+1}=\alpha_{n} f\left(x_{n}\right)+\left(1-\alpha_{n}\right) z_{n},
\end{array}\right.
$$


where the operator $A$ is monotone and Lipschitz continuous, $f$ is a strict contraction mapping, $l \in(0,1), \mu \in(0,1)$, and $\lambda_{n}=l^{m_{n}}$ where $m_{n}$ is the smallest nonnegative integer $m$ such that

$$
\lambda\left\|A x_{n}-A y_{n}\right\| \leq \mu\left\|r_{l^{m_{n}}}\left(x_{n}\right)\right\|
$$

where $r_{l^{m_{n}}}(x)=x-P_{C}\left(x-l^{m_{n}} A(x)\right)$ for all $x \in C$. Recently, the sequence produced by the following algorithm was introduced by Thong and Hieu [3] based on Tseng's method (THEGM):

$$
\left\{\begin{array}{l}
y_{n}=P_{C}\left(x_{n}-\lambda_{n} A x_{n}\right), \\
z_{n}=y_{n}-\lambda_{n}\left(A y_{n}-A x_{n}\right), \\
x_{n+1}=\alpha_{n} f\left(x_{n}\right)+\left(1-\alpha_{n}\right) z_{n},
\end{array}\right.
$$

where the operator $A$ is monotone and Lipschitz continuous, $\gamma>0, l \in(0,1), \mu \in(0,1)$, and $\lambda_{n}$ is chosen to be the largest $\lambda \in\left\{\gamma, \gamma l, \gamma l^{2}, \cdots\right\}$ satisfying

$$
\lambda\left\|A x_{n}-A y_{n}\right\| \leq \mu\left\|x_{n}-y_{n}\right\|
$$

In this paper, substituting a sequence $\left\{\beta_{n}\right\} \subset(0,1)$ of coefficients instead of the sequence $\left\{1-\alpha_{n}\right\}$ in the algorithm (6), we extend algorithm (6). Moreover, condition (7) will be removed just by a slight change in the coefficients $\left\{\lambda_{n}\right\}$. Also, a sequence of computational errors in our algorithm is considered. The strong convergence of the proposed algorithm to a point of the variational inequality $\operatorname{VI}(C, A)$ will be proved under the presence of computational errors. Finally, some examples will be presented which will examine the convergence of the proposed algorithm in different situations.

\section{Preliminaries}

In this section, some basic concepts are presented.

Let $H$ be a real Hilbert space with the inner product $\langle.$, . $\rangle$ and norm $\|$.$\| and suppose that C$ is a nonempty closed convex subset of $H$ and $A: C \longrightarrow H$ is an operator. The operator $A$ is said to be

(i) Monotone if

$$
\langle A x-A y, x-y\rangle \geq 0, \forall x, y \in C,
$$

(ii) $L$-Lipchitz continuous if there exist $L>0$ such that

$$
\|A x-A y\| \leq L\|x-y\|, \forall x, y \in C \text {. }
$$

For the main results of this paper, we need the following useful lemmas.
Lemma 1. Let $H$ be a real Hilbert space. Then, we have the following well-known results:

$$
\begin{aligned}
& \|x+y\|^{2}=\|x\|^{2}+2\langle x, y\rangle+\|y\|^{2}, \forall x, y \in H . \\
& \|x+y\|^{2} \leq\|x\|^{2}+2\langle y, x+y\rangle, \forall x, y \in H .
\end{aligned}
$$

Lemma 2 (Xu, see [16]). Let $\left\{a_{n}\right\}$ be a sequence of nonnegative real numbers satisfying the following relation:

$$
a_{n+1} \leq\left(1-\eta_{n}\right) a_{n}+\eta_{n} \sigma_{n}+\gamma_{n}, n \geq 1,
$$

where

(a) $\left\{\eta_{n}\right\} \subset[0,1], \sum_{n=1}^{\infty} \eta_{n}=\infty$

(b) $\lim \sup \sigma_{n} \leq 0$

(c) $\gamma_{n} \geq 0(n \geq 1), \sum_{n=1}^{\infty} \gamma_{n}<\infty$

Then, $a_{n} \longrightarrow 0$ as $n \longrightarrow \infty$.

Lemma 3 (see [17]). Let $C$ be a closed and convex subset in a real Hilbert space $H$. Then, $z=P_{C} x$ if and only if $\langle x-z, y-z\rangle$ $\leq 0 \forall y \in C$.

Lemma 4 (see [18]). Let $\left\{a_{n}\right\}$ be a sequence of nonnegative real numbers such that there exists a subsequence $\left\{a_{n_{j}}\right\}$ of $\left\{a_{n}\right\}$ such that $a_{n_{j}}<a_{n_{j}+1}$ for all $j \in \mathbb{N}$. Then, there exists a nondecreasing sequence $\left\{m_{k}\right\}$ of $\mathbb{N}$ such that $\lim _{k \rightarrow \infty}$ $m_{k}=\infty$ and the following properties are satisfied by all (sufficiently large) number $k \in \mathbb{N}$ :

$$
a_{m_{k}} \leq a_{m_{k}+1} \text { and } a_{k} \leq a_{m_{k}+1}
$$

In fact, $m_{k}$ is the largest number $n$ in the set $\{1,2, \cdots, k\}$ such that $a_{n}<a_{n+1}$.

Lemma 5 (see [3]). Let $\left\{x_{n}\right\}$ be a sequence generated by algorithm (3). Then,

$$
\left\|x_{n+1}-p\right\|^{2} \leq\left\|x_{n}-p\right\|^{2}-\left(1-\mu^{2}\right)\left\|x_{n}-y_{n}\right\| \forall p \in V I(C, A) .
$$

\section{Main Results}

In this section, we prove a strong convergence theorem for finding a common element of the set of solutions of an equilibrium problem and a fixed point problem.

Theorem 6. Let $C$ be a nonempty closed convex subset of a real Hilbert space $H, A$ be a monotone, and L-Lipschitz continuous mapping on $C$ and $\lambda \in(0,1)$ such that $\lambda L<1$. Suppose that $f: H \longrightarrow H$ is a contraction mapping with a constant $\rho \in[0,1)$. Let $\left\{e_{n}\right\} \subseteq H$ be a sequence of computational errors, $x_{0} \in H$ be arbitrary and $\left\{x_{n}\right\},\left\{y_{n}\right\}$, and $\left\{z_{n}\right\}$ be the sequences generated by 


$$
\left\{\begin{array}{l}
y_{n}=P_{C}\left(x_{n}-\lambda A x_{n}\right), \\
z_{n}=y_{n}-\lambda\left(A y_{n}-A x_{n}\right), \\
x_{n+1}=\alpha_{n} f\left(x_{n}\right)+\beta_{n} z_{n}+e_{n},
\end{array}\right.
$$

where $\left\{\alpha_{n}\right\}$ and $\left\{\beta_{n}\right\}$ are real sequences in $[0,1]$ such that $\alpha_{n}$ $+\beta_{n} \leq 1$ for each $n \geq 1$. Also, assume the following conditions:

$$
\begin{aligned}
& \sum_{n=1}^{\infty} \alpha_{n} \beta_{n}=\infty, \\
& \sum_{n=1}^{\infty}\left(1-\alpha_{n}-\beta_{n}\right)<\infty, \\
& \lim _{n \longrightarrow \infty} \frac{\left(1-\alpha_{n}-\beta_{n}\right)}{\alpha_{n}}=\lim _{n \longrightarrow \infty} \alpha_{n}=0, \\
& \sum_{n=1}^{\infty}\left\|e_{n}\right\|<\infty .
\end{aligned}
$$

Then,

(i) $\operatorname{VI}(C, A) \neq \varnothing$ if and only if $\left\{x_{n}\right\}$ is bounded and $\liminf _{n \rightarrow \infty}\left\|x_{n}-y_{n}\right\|=0$

Suppose $\operatorname{VI}(C, A) \neq \varnothing$. Then,

(ii) If $\lim _{n \rightarrow \infty}\left\|e_{n}\right\| / \alpha_{n}=0$, then $\left\{x_{n}\right\}$ converges strongly to $q=P_{V I(C, A)} \circ f(q)$, where $P_{V I(C, A)} \circ f: H \longrightarrow V I(C$, $A)$ is the mapping defined by $P_{V I(C, A)} \circ f(x)=$ $P_{V I(C, A)}(f(x))$ for each $x \in H$

Proof. (i) Assume that $\left\{x_{n}\right\}$ is a bounded sequence and $\liminf _{n \rightarrow \infty}\left\|x_{n}-y_{n}\right\|=0$. Then, there exists a subsequence $\left\{n_{i}\right\} \subset \mathbb{N}$ such that $\left\|x_{n_{i}}-y_{n_{i}}\right\| \longrightarrow 0$ when $i \longrightarrow \infty$. Note $\left\{x_{n_{i}}\right\}$ is a bounded sequence. Hence, there exists a subsequence $\left\{x_{n_{i_{k}}}\right\}$ of $\left\{x_{n_{i}}\right\}$ such that $\left\{x_{n_{i_{k}}}\right\}$ converges weakly to some $x \in C$. Now, noting that since $y_{n_{i_{k}}}=P_{C}\left(x_{n_{i_{k}}}-\lambda A x_{n_{i_{k}}}\right)$, for all $z \in C$, we have

$$
\begin{aligned}
0 \geq & \left\langle x_{n_{i_{k}}}-\lambda A x_{n_{i_{k}}}-y_{n_{i_{k}}}, z-y_{n_{i_{k}}}\right\rangle \\
= & \left\langle x_{n_{i_{k}}}-y_{n_{i_{k}}}, z-y_{n_{i_{k}}}\right\rangle-\lambda\left\langle A x_{n_{i_{k}}}, z-y_{n_{i_{k}}}\right\rangle \\
= & \left\langle x_{n_{i_{k}}}-y_{n_{i_{k}}}, z-y_{n_{i_{k}}}\right\rangle-\lambda\left\langle A x_{n_{i_{k}}}, z-x_{n_{i_{k}}}\right\rangle-\lambda\left\langle A x_{n_{i_{k}}}, x_{n_{i_{k}}}-y_{n_{i_{k}}}\right\rangle \\
= & \left\langle x_{n_{i_{k}}}-y_{n_{i_{k}}}, z-y_{n_{i_{k}}}\right\rangle-\lambda\left\langle A x_{n_{i_{k}}}-A z, z-x_{n_{i_{k}}}\right\rangle \\
& -\lambda\left\langle A z, z-x_{n_{i_{k}}}\right\rangle-\lambda\left\langle A x_{n_{i_{k}}}, x_{n_{i_{k}}}-y_{n_{i_{k}}}\right\rangle \\
\geq & \left\langle x_{n_{i_{k}}}-y_{n_{i_{k}}}, z-y_{n_{i_{k}}}\right\rangle-\lambda\left\langle A z, z-x_{n_{i_{k}}}\right\rangle \\
& -\lambda\left\langle A x_{n_{i_{k}}}, x_{n_{i_{k}}}-y_{n_{i_{k}}}\right\rangle .
\end{aligned}
$$

Now, we have

$$
-\lambda\left\langle A z, z-x_{n_{i_{k}}}\right\rangle \leq\left\langle x_{n_{i_{k}}}-y_{n_{i_{k}}}, y_{n_{i_{k}}}-z\right\rangle+\lambda\left\langle A x_{n_{i_{k}}}, x_{n_{i_{k}}}-y_{n_{i_{k}}}\right\rangle .
$$

Therefore,

$$
-\lambda\left\langle A z, z-x_{n_{i_{k}}}\right\rangle \leq\left\|y_{n_{i_{k}}}-x_{n_{i_{k}}}\right\|\left\|y_{n_{i_{k}}}-z\right\|+\lambda\left\|A x_{n_{i_{k}}}\right\|\left\|x_{n_{i_{k}}}-y_{n_{i_{k}}}\right\| .
$$

From $\lim _{k \longrightarrow \infty}\left\|x_{n_{i_{k}}}-y_{n_{i_{k}}}\right\|=0$, we have $-\lambda\langle A z, z-x\rangle \leq 0$ for all $z \in C$. Now, let $y \in C$ and $0<t<1$, and from the convexity $C$ we have $y_{t}=[t y+(1-t) x] \in C$. Therefore,

$$
0 \leq\left\langle A y_{t}, y_{t}-x\right\rangle=\left\langle A y_{t}, t y-t x\right\rangle=t\left\langle A y_{t}, y-x\right\rangle
$$

Since $0<t<1$ then $\left\langle A y_{t}, y-x\right\rangle \geq 0$ for all $y \in C$. Because the mapping $A$ and multiplication are continuous, if $t \longrightarrow 0$, then we have $\langle A x, y-x\rangle \geq 0$ for all $y \in C$, i.e; $x \in \operatorname{VI}(C, A)$.

For the converse fix $p \in \operatorname{VI}(C, A)$, using Lemma 5, we have

$$
\left\|z_{n}-p\right\|^{2} \leq\left\|x_{n}-p\right\|^{2}-\left(1-(\lambda L)^{2}\right)\left\|x_{n}-y_{n}\right\|^{2} .
$$

Therefore,

$$
\left\|z_{n}-p\right\| \leq\left\|x_{n}-p\right\|
$$

Using the above inequality, we have

$$
\begin{aligned}
1\left\|x_{n+1}-p\right\|= & \left\|\alpha_{n} f\left(x_{n}\right)+\beta_{n} z_{n}+e_{n}-p\right\| \\
= & \left\|\alpha_{n}\left(f\left(x_{n}\right)-p\right)+\beta_{n}\left(z_{n}-p\right)-\left(1-\alpha_{n}-\beta_{n}\right) p+e_{n}\right\| \\
\leq & \alpha_{n}\left\|f\left(x_{n}\right)-p\right\|+\beta_{n}\left\|z_{n}-p\right\|+\left(1-\alpha_{n}-\beta_{n}\right)\|p\|+\left\|e_{n}\right\| \\
\leq & \alpha_{n}\left\|f\left(x_{n}\right)-f(p)\right\|+\alpha_{n}\|f(p)-p\| \\
& +\beta_{n}\left\|x_{n}-p\right\|+\left(1-\alpha_{n}-\beta_{n}\right)\|p\|+\left\|e_{n}\right\| \\
\leq & \alpha_{n} \rho\left\|x_{n}-p\right\|+\alpha_{n}\|f(p)-p\|+\beta_{n}\left\|x_{n}-p\right\| \\
& +\left(1-\alpha_{n}-\beta_{n}\right)\|p\|+\left\|e_{n}\right\| \\
= & \left(\alpha_{n} \rho+\beta_{n}\right)\left\|x_{n}-p\right\|+\alpha_{n}(1-\rho) \frac{\|f(p)-p\|}{1-\rho} \\
& +\left(1-\alpha_{n}-\beta_{n}\right)\|p\|+\left\|e_{n}\right\| \\
\leq & \max \left\{\left\|x_{n}-p\right\|, \frac{\|f(p)-p\|}{1-\rho},\|p\|\right\}+\left\|e_{n}\right\|,
\end{aligned}
$$

so the sequence $\left\{x_{n}\right\}$ is bounded.

(ii) Let $p \in \operatorname{VI}(C, A)$. From part (i), we have that $\left\{x_{n}\right\}$ is bounded. Then $\left\{f\left(x_{n}\right)\right\},\left\{y_{n}\right\}$ and $\left\{z_{n}\right\}$ are bounded. Now, using Lemma 5, we have

$$
\left\|z_{n}-p\right\|^{2} \leq\left\|x_{n}-p\right\|^{2}-\left(1-(\lambda L)^{2}\right)\left\|x_{n}-y_{n}\right\|^{2} \forall p \in \operatorname{VI}(C, A),
$$

(note that our sequence $\left\{z_{n}\right\}$ in the algorithm (14) replaces $\left\{x_{n+1}\right\}$ in Lemma 5).

Since $\alpha_{n}+\beta_{n}+\left(1-\alpha_{n}-\beta_{n}\right)=1$, by the convexity of $\|.\|^{2}$ and the relation $\|x+y\|^{2}=\|x\|^{2}+2\langle x, y\rangle+\|y\|^{2}$, we conclude that 


$$
\begin{aligned}
\left\|x_{n+1}-p\right\|^{2}= & \left\|\alpha_{n} f\left(x_{n}\right)+\beta_{n} z_{n}+e_{n}-p\right\|^{2} \\
= & \left\|\alpha_{n}\left(f\left(x_{n}\right)+e_{n}-p\right)+\beta_{n}\left(z_{n}+e_{n}-p\right)+\left(1-\alpha_{n}-\beta_{n}\right)\left(e_{n}-p\right)\right\|^{2} \\
\leq & \alpha_{n}\left\|f\left(x_{n}\right)+e_{n}-p\right\|^{2}+\beta_{n}\left\|z_{n}+e_{n}-p\right\|^{2}+\left(1-\alpha_{n}-\beta_{n}\right)\left\|e_{n}-p\right\|^{2} \\
\leq & \left.\alpha_{n}\left\|f\left(x_{n}\right)-p\right\|^{2}+\left(1-\alpha_{n}\right)\left\|z_{n}-p\right\|^{2}+\left(1-\alpha_{n}-\beta_{n}\right)\right)\|p\|^{2} \\
& +\left(\alpha_{n}+\beta_{n}+\left(1-\alpha_{n}-\beta_{n}\right)\right)\left\|e_{n}\right\|^{2} \\
& +2\left\langle\alpha_{n} f\left(x_{n}\right)-\alpha_{n} p+\beta_{n} z_{n}-\beta_{n} p-\left(1-\alpha_{n}-\beta_{n}\right) p, e_{n}\right\rangle \\
\leq & \alpha_{n}\left\|f\left(x_{n}\right)-p\right\|^{2}+\left(1-\alpha_{n}\right)\left\|x_{n}-p\right\|^{2} \\
& -\left(1-\alpha_{n}\right)\left(1-(\lambda L)^{2}\right)\left\|x_{n}-y_{n}\right\|^{2}+\left(1-\alpha_{n}-\beta_{n}\right)\|p\|^{2} \\
& +\left\|e_{n}\right\|^{2}+2\left\langle\alpha_{n} f\left(x_{n}\right)+\beta_{n} z_{n}-p, e_{n}\right\rangle(b y(3.3)) \\
\leq & \alpha_{n}\left\|f\left(x_{n}\right)-p\right\|^{2}+\left\|x_{n}-p\right\|^{2}-\left(1-\alpha_{n}\right)\left(1-(\lambda L)^{2}\right)\left\|x_{n}-y_{n}\right\|^{2} \\
& +\left(1-\alpha_{n}-\beta_{n}\right)\|p\|^{2}+\left\|e_{n}\right\|^{2}+2\left\|\alpha_{n} f\left(x_{n}\right)+\beta_{n} z_{n}-p\right\|\left\|e_{n}\right\| .
\end{aligned}
$$

Therefore,

$$
\begin{aligned}
& \left(1-\alpha_{n}\right)\left(1-(\lambda L)^{2}\right)\left\|x_{n}-y_{n}\right\|^{2} \leq\left\|x_{n}-p\right\|^{2}-\left\|x_{n+1}-p\right\|^{2} \\
& \quad+\alpha_{n}\left\|f\left(x_{n}\right)-p\right\|^{2}+\left(1-\alpha_{n}-\beta_{n}\right)\|p\|^{2}+\left\|e_{n}\right\|^{2} \\
& \quad+2\left\|\alpha_{n} f\left(x_{n}\right)+\beta_{n} z_{n}-p\right\|\left\|e_{n}\right\| .
\end{aligned}
$$

Also, we have,

$$
\begin{aligned}
\left\|x_{n+1}-x_{n}\right\|= & \left\|\alpha_{n} f\left(x_{n}\right)+\beta_{n} z_{n}+e_{n}-x_{n}\right\| \\
= & \left\|\alpha_{n}\left(f\left(x_{n}\right)-x_{n}\right)+\beta_{n}\left(z_{n}-x_{n}\right)-\left(1-\alpha_{n}-\beta_{n}\right) x_{n}+e_{n}\right\| \\
\leq & \alpha_{n}\left\|f\left(x_{n}\right)-x_{n}\right\|+\beta_{n}\left\|z_{n}-x_{n}\right\|+\left(1-\alpha_{n}-\beta_{n}\right)\left\|x_{n}\right\|+\left\|e_{n}\right\| \\
= & \alpha_{n}\left\|f\left(x_{n}\right)-x_{n}\right\|+\beta_{n}\left\|y_{n}-\lambda\left(A y_{n}-A x_{n}\right)-x_{n}\right\| \\
& +\left(1-\alpha_{n}-\beta_{n}\right)\left\|x_{n}\right\|+\left\|e_{n}\right\| \leq \alpha_{n}\left\|f\left(x_{n}\right)-x_{n}\right\| \\
& +\beta_{n}\left\|y_{n}-x_{n}\right\|+\beta_{n} \lambda L\left\|y_{n}-x_{n}\right\| \\
& +\left(1-\alpha_{n}-\beta_{n}\right)\left\|x_{n}\right\|+\left\|e_{n}\right\| .
\end{aligned}
$$

Note that $P_{\mathrm{VI}(C, A)} \circ f$ is a contraction mapping. Then, by the Banach contraction principle, there exists a unique element $q \in H$ such that $q=P_{\mathrm{VI}(C, A)} \circ f(q)$. Now, we claim that $\left\{x_{n}\right\}$ converges strongly to $q=P_{\mathrm{VI}(C, A)} \circ f(q)$. It is enough to consider two cases:

Case 1. Suppose there exists some $n_{0} \in \mathbb{N}$ such that $\| x_{n+1}-$ $q\left\|^{2} \leq\right\| x_{n}-q \|^{2}$ for all $n \geq n_{0}$. Then, $\lim _{n \longrightarrow \infty}\left\|x_{n}-q\right\|$ exists. From (25) and our assumptions, $\lim _{n \rightarrow \infty}\left\|x_{n}-y_{n}\right\|=0$. From the conditions (b), (c), and (d), $\lim _{n \rightarrow \infty}\left(1-\alpha_{n}-\beta_{n}\right)=\lim _{n \rightarrow \infty}$ $\alpha_{n}=\lim _{n \rightarrow \infty}\left\|e_{n}\right\|=0$. Then, from (26) and the boundedness of the sequences $\left\{\left\|x_{n}\right\|\right\},\left\{\left\|f\left(x_{n}\right)-x_{n}\right\|\right\}$ and $\left\{\beta_{n}\right\}$, we obtain that

$$
\lim _{n \longrightarrow \infty}\left\|x_{n+1}-x_{n}\right\|=0 \text {. }
$$

Now, we have

$\left\|x_{n+p}-x_{n}\right\| \leq\left\|x_{n+p}-x_{n+p-1}\right\|+\left\|x_{n+p-1}-x_{n+p-2}\right\|+\cdots+\left\|x_{n+1}-x_{n}\right\|$,

for all $p \in \mathbb{N}$. Note that from (27), it is concluded that $\lim _{n \rightarrow \infty} \|$ $x_{n+p-i}-x_{n+p-(i+1)} \|=0$, for all $0 \leq i \leq p-1$ and $p \in \mathbb{N}$. Then, from (28), $\lim _{n \rightarrow \infty}\left\|x_{n+p}-x_{n}\right\|=0$, for all $p \in \mathbb{N}$, i.e., $\left\{x_{n}\right\}$ is a Cauchy sequence in the Hilbert space $H$; therefore, $\left\{x_{n}\right\}$ is convergent. Now we show that $\left\{x_{n}\right\}$ converges strongly to $q$. Note that

$$
\begin{aligned}
\left\|x_{n+1}-q\right\|^{2}= & \left\|\alpha_{n} f\left(x_{n}\right)+\beta_{n} z_{n}+e_{n}-q\right\|^{2} \\
= & \| \alpha_{n}\left(f\left(x_{n}\right)+e_{n}-q\right)+\beta_{n}\left(z_{n}+e_{n}-q\right) \\
& +\left(1-\alpha_{n}-\beta_{n}\right)\left(e_{n}-q\right) \|^{2} \\
\leq & \beta_{n}^{2}\left\|z_{n}+e_{n}-q\right\|^{2}+2\left\langle\alpha_{n}\left(f\left(x_{n}\right)+e_{n}-q\right)\right. \\
& \left.+\left(1-\alpha_{n}-\beta_{n}\right)\left(e_{n}-q\right), x_{n+1}-q\right\rangle(\text { by Lemma } 2.1) \\
= & \beta_{n}^{2}\left\|z_{n}+e_{n}-q\right\|^{2}+2 \alpha_{n}\left\langle\left(f\left(x_{n}\right)+e_{n}-q\right), x_{n+1}-q\right\rangle \\
& +2\left(1-\alpha_{n}-\beta_{n}\right)\left\langle e_{n}-q, x_{n+1}-q\right\rangle \\
= & \beta_{n}^{2}\left\|z_{n}+e_{n}-q\right\|^{2}+2 \alpha_{n}\left\langle\left(f\left(x_{n}\right)-q\right), x_{n+1}-q\right\rangle \\
& +2 \alpha_{n}\left\langle e_{n}, x_{n+1}-q\right\rangle+2\left(1-\alpha_{n}-\beta_{n}\right)\left\langle e_{n}-q, x_{n+1}-q\right\rangle \\
\leq & \beta_{n}^{2}\left\|z_{n}+e_{n}-q\right\|^{2}+2 \alpha_{n} \rho\left\|x_{n}-q\right\|\left\|x_{n+1}-q\right\| \\
& +2 \alpha_{n}\left\langle f(q)-q, x_{n+1}-q\right\rangle+2 \alpha_{n}\left\langle e_{n}, x_{n+1}-q\right\rangle \\
& +2\left(1-\alpha_{n}-\beta_{n}\right)\left\langle e_{n}-q, x_{n+1}-q\right\rangle \leq \beta_{n}^{2}\left\|z_{n}-q\right\|^{2} \\
& +2 \beta_{n}^{2}\left\langle e_{n}, z_{n}+e_{n}-q\right\rangle+2 \alpha_{n} \rho\left\|x_{n}-q\right\|^{2}(\text { by Lemma } 2.1) \\
& +2 \alpha_{n}\left\langle f(q)-q, x_{n+1}-q\right\rangle+2 \alpha_{n}\left\langle e_{n}, x_{n+1}-q\right\rangle \\
& +2\left(1-\alpha_{n}-\beta_{n}\right)\left\langle e_{n}-q, x_{n+1}-q\right\rangle \\
\leq & \left(1-\alpha_{n}\right)^{2}\left\|x_{n}-q\right\|^{2}+2 \alpha_{n} \rho\left\|x_{n}-q\right\|^{2} \\
& +2 \alpha_{n}\left\langle f(q)-q, x_{n+1}-q\right\rangle+2 \beta_{n}^{2}\left\langle e_{n}, z_{n}+e_{n}-q\right\rangle \\
& +2 \alpha_{n}\left\langle e_{n}, x_{n+1}-q\right\rangle+2\left(1-\alpha_{n}-\beta_{n}\right)\left\langle e_{n}-q, x_{n+1}-q\right\rangle \\
= & \left(1-2 \alpha_{n}(1-\rho)\right)\left\|x_{n}-q\right\|^{2}+2 \alpha_{n}(1-\rho) \\
& +\left[\frac{\alpha_{n}\left\|x_{n}-q\right\|^{2}}{2(1-\rho)}+\frac{\left\langle f(q)-q, x_{n+1}-q\right\rangle}{1-\rho}\right] \\
& +2 \beta_{n}^{2}\left\langle e_{n}, z_{n}+e_{n}-q\right\rangle+2 \alpha_{n}\left\langle e_{n}, x_{n+1}-q\right\rangle \\
& +2\left(1-\alpha_{n}-\beta_{n}\right)\left\langle e_{n}-q, x_{n+1}-q\right\rangle \\
\leq & \left(1-2 \alpha_{n}(1-\rho)\right)\left\|x_{n}-q\right\|^{2}+2 \alpha_{n}(1-\rho) \\
& +\left[\frac{\alpha_{n}\left\|x_{n}-q\right\|^{2}}{2(1-\rho)}+\frac{\left\langle f(q)-q, x_{n+1}-q\right\rangle}{1-\rho}\right] \\
& +2 \beta_{n}^{2}\left\|e_{n}\right\|\left\|z_{n}+e_{n}-q\right\|+2 \alpha_{n}\left\|e_{n}\right\|\left\|x_{n+1}-q\right\| \\
& +2\left(1-\alpha_{n}-\beta_{n}\right)\left\|e_{n}-q\right\|\left\|x_{n+1}-q\right\| . \\
& +2, q) \\
&
\end{aligned}
$$

Since $\left\{x_{n}\right\}$ is strongly convergent, so $x_{n} \longrightarrow z$ for some $z \in C$, then we conclude that $x_{n} \rightarrow z$. Also, as in the proof of part (i), we conclude $z \in \operatorname{VI}(C, A)$. Therefore, from Lemma 3

$$
\limsup _{n \rightarrow \infty}\left\langle f(q)-q, x_{n+1}-q\right\rangle=\langle f(q)-q, z-q\rangle \leq 0 .
$$

Next, we consider the sequences $\left\{\eta_{n}\right\},\left\{a_{n}\right\},\left\{\sigma_{n}\right\}$ and $\left\{\gamma_{n}\right\}$ in (3.8) as follows:

$$
\begin{aligned}
\eta_{n}:= & 2 \alpha_{n}(1-\rho), \\
a_{n}:= & \left\|x_{n}-q\right\|^{2}, \\
\sigma_{n}:= & \frac{\alpha_{n}\left\|x_{n}-q\right\|^{2}}{2(1-\rho)}+\frac{\left\langle f(q)-q, x_{n+1}-q\right\rangle}{1-\rho}, \\
\gamma_{n}:= & 2 \beta_{n}^{2}\left\|e_{n}\right\|\left\|z_{n}+e_{n}-q\right\|+2 \alpha_{n}\left\|e_{n}\right\|\left\|x_{n+1}-q\right\| \\
& +2\left(1-\alpha_{n}-\beta_{n}\right)\left\|e_{n}-q\right\|\left\|x_{n+1}-q\right\| .
\end{aligned}
$$




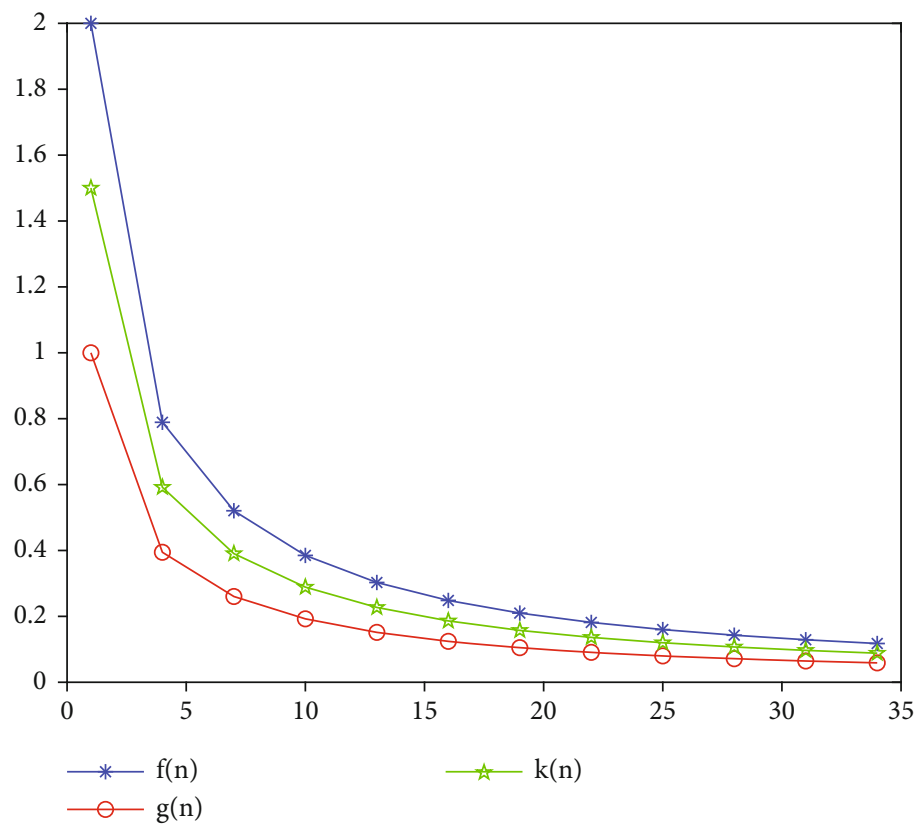

Figure 1: Example 1. Convergence behavior $\left\{f_{n}\right\}$ in Example 1.

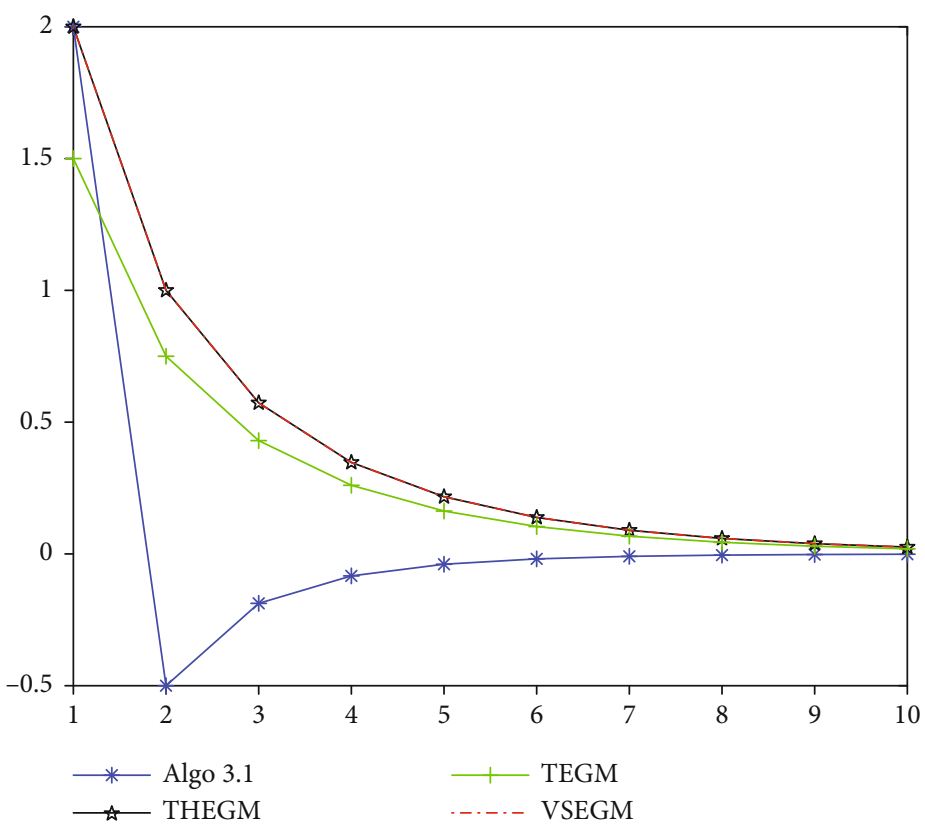

FIGURE 2: The number of iteration for convergence to zero is, respectively, 15, 27, 25, and 27 in Example 2.

From the above, we have

$$
a_{n+1} \leq\left(1-\eta_{n}\right) a_{n}+\eta_{n} \sigma_{n}+\gamma_{n}
$$

From $(c)$, there exists an integer $n_{1} \in \mathbb{N}$ such that $\left\{\eta_{n}\right\} \subset[0,1]$, for each $n \geq n_{1}$. Without loss of generality, we may assume that $\left\{\eta_{n}\right\} \subset[0,1]$, for each $n \geq 1$. From condition (a), note $\infty=\sum_{n=1}^{\infty} \alpha_{n} \beta_{n} \leq \sum_{n=1}^{\infty} \alpha_{n}$, so $\sum_{n=1}^{\infty} \alpha_{n}=\infty$, and hence, $\sum_{n=1}^{\infty} \eta_{n}=2(1-\rho) \sum_{n=1}^{\infty} \alpha_{n}=\infty$. Therefore, condition $(a)$ of
Lemma 2 holds. From $(c)$, the boundedness of $\left\|x_{n}-q\right\|^{2}$ and (3.9), we have

$$
\begin{aligned}
\limsup _{n \longrightarrow \infty} \sigma_{n} & =\limsup _{n \longrightarrow \infty}\left(\frac{\alpha_{n}\left\|x_{n}-q\right\|^{2}}{2(1-\rho)}+\frac{\left\langle f(q)-q, x_{n+1}-q\right\rangle}{1-\rho}\right) \\
& \leq \limsup _{n \rightarrow \infty} \frac{\alpha_{n}\left\|x_{n}-q\right\|^{2}}{2(1-\rho)}+\limsup _{n \rightarrow \infty} \frac{\left\langle f(q)-q, x_{n+1}-q\right\rangle}{1-\rho} \\
& \leq 0 .
\end{aligned}
$$


Therefore, condition $(b)$ of Lemma 2 holds. Next, note $\gamma_{n} \geq 0$ and $\left\|z_{n}+e_{n}-q\right\|,\left\|x_{n+1}-q\right\|$, and $\left\|e_{n}-q\right\|$ are bounded. Hence, there exist some positive constants $M_{1}$, $M_{2}$, and $M_{3}$ such that, $\left\|z_{n}+e_{n}-q\right\| \leq M_{1},\left\|x_{n+1}-q\right\| \leq M_{2}$ and $\left\|e_{n}-q\right\| \leq M_{3}$, for each $n \geq 1$. Then, from $(b)$ and $(d)$, we have

$$
\begin{aligned}
\sum_{n=1}^{\infty} \gamma_{n}= & \sum_{n=1}^{\infty}\left[2 \beta_{n}^{2}\left\|e_{n}\right\|\left\|z_{n}+e_{n}-q\right\|+2 \alpha_{n}\left\|e_{n}\right\|\left\|x_{n+1}-q\right\|\right. \\
& \left.+2\left(1-\alpha_{n}-\beta_{n}\right)\left\|e_{n}-q\right\|\left\|x_{n+1}-q\right\|\right] \\
\leq & \Sigma_{n=1}^{\infty}\left[2 \beta_{n}^{2}\left\|e_{n}\right\| M_{1}+2 \alpha_{n}\left\|e_{n}\right\| M_{2}+2\left(1-\alpha_{n}-\beta_{n}\right) M_{3} M_{2}\right] \\
\leq & 2 M_{1} \Sigma_{n=1}^{\infty}\left\|e_{n}\right\|+2 M_{2} \Sigma_{n=0}^{\infty}\left\|e_{n}\right\|+2 M_{3} M_{2} \Sigma_{n=1}^{\infty}\left(1-\alpha_{n}-\beta_{n}\right) \\
& <\infty .
\end{aligned}
$$

Therefore, condition $(c)$ of Lemma 2 holds. Then, from Lemma 2 , it follows that $a_{n} \longrightarrow 0$ as $n \longrightarrow \infty$, i.e., $\lim _{n \longrightarrow \infty}$ $\left\|x_{n}-q\right\|^{2}=0$.

Case 2. Suppose there exists a subsequence $\left\{\left\|x_{n_{j}}-q\right\|^{2}\right\}$ of $\left\{\left\|x_{n}-q\right\|^{2}\right\}$ such that $\left\|x_{n_{j}}-q\right\|^{2}<\left\|x_{n_{j}+1}-q\right\|^{2}$ for all $j \in \mathbb{N}$. Now from Lemma 4 , there exists a nondecreasing sequence $\left\{m_{k}\right\}$ of $\mathbb{N}$ such that $\lim _{k \rightarrow \infty} m_{k}=\infty$ and the following inequalities hold for all $k \in \mathbb{N}$ :

$$
\left\|x_{m_{k}}-q\right\|^{2} \leq\left\|x_{m_{k}+1}-q\right\|^{2} \text { and }\left\|x_{k}-q\right\|^{2} \leq\left\|x_{m_{k}+1}-q\right\|^{2} .
$$

Now, from (25), we have

$$
\begin{aligned}
(1- & \left.\alpha_{\mathrm{m}_{k}}\right)\left(1-(\lambda L)^{2}\right)\left\|x_{m_{k}}-y_{m_{k}}\right\|^{2} \\
\leq & \left\|x_{m_{k}}-p\right\|^{2}-\left\|x_{m_{k}+1}-p\right\|^{2}+\alpha_{m_{k}}\left\|f\left(x_{m_{k}}\right)-p\right\|^{2} \\
& +\left(1-\alpha_{m_{k}}-\beta_{m_{k}}\right)\|p\|^{2}+\left\|e_{m_{k}}\right\|^{2} \\
& +2\left\|\alpha_{m_{k}} f\left(x_{m_{k}}\right)+\beta_{m_{k}} z_{m_{k}}-p\right\|\left\|e_{m_{k}}\right\| .
\end{aligned}
$$

Hence, $\lim _{k \longrightarrow \infty}\left\|x_{m_{k}}-y_{m_{k}}\right\|=0$, therefore from (26) (adjusted) $\lim _{k \longrightarrow \infty}\left\|x_{m_{k}+1}-x_{m_{k}}\right\|=0$.

From (29) (adjusted), we conclude that

$$
\begin{aligned}
\left\|x_{m_{k}+1}-q\right\|^{2} \leq & \left(1-2 \alpha_{m_{k}}(1-\rho)\right)\left\|x_{m_{k}}-q\right\|^{2}+2 \alpha_{m_{k}}(1-\rho) \\
& \cdot\left[\frac{\alpha_{m_{k}}\left\|x_{m_{k}}-q\right\|^{2}}{2(1-\rho)}+\frac{\left\langle f(q)-q, x_{m_{k}+1}-q\right\rangle}{1-\rho}\right] \\
& +2 \beta_{m_{k}}^{2}\left\|e_{m_{k}}\right\|\left\|z_{m_{k}}+e_{m_{k}}-q\right\|+2 \alpha_{m_{k}}\left\|e_{m_{k}}\right\|\left\|x_{m_{k}+1}-q\right\| \\
& +2\left(1-\alpha_{m_{k}}-\beta_{m_{k}}\right)\left\|e_{m_{k}}-q\right\|\left\|x_{m_{k}+1}-q\right\| \\
\leq & \left(1-2 \alpha_{m_{k}}(1-\rho)\right)\left\|x_{m_{k}+1}-q\right\|^{2}+2 \alpha_{m_{k}}(1-\rho) \\
& \cdot\left[\frac{\alpha_{m_{k}}\left\|x_{m_{k}}-q\right\|^{2}}{2(1-\rho)}+\frac{\left\langle f(q)-q, x_{m_{k}+1}-q\right\rangle}{1-\rho}\right] \\
& +2 \beta_{m_{k}}^{2}\left\|e_{m_{k}}\right\|\left\|z_{m_{k}}+e_{m_{k}}-q\right\| \\
& +2 \alpha_{m_{k}}\left\|e_{m_{k}}\right\|\left\|x_{m_{k}+1}-q\right\| \\
& +2\left(1-\alpha_{m_{k}}-\beta_{m_{k}}\right)\left\|e_{m_{k}}-q\right\|\left\|x_{m_{k}+1}-q\right\| .
\end{aligned}
$$

TABLE 1: Numerical results of convergence for $x_{1}=2$ in Example 3 .

\begin{tabular}{lccc}
\hline$n$ & $x_{n}$ & $y_{n}$ & $z_{n}$ \\
\hline 1 & 2 & 1 & 1.5 \\
2 & 0.5 & 0.25 & 0.375 \\
3 & 0.4429 & 0.2214 & 0.3321 \\
93 & 0.0005 & 0.0002 & 0.0003 \\
94 & 0.0005 & 0.0002 & 0.0003 \\
95 & 0.0004 & 0.0002 & 0.0003 \\
185 & 0.0001 & 0.0001 & 0.0001 \\
186 & 0.0001 & 0.0001 & 0.0001 \\
187 & 0.0001 & 0.0001 & 0.0001 \\
277 & 0.0001 & 0.0000 & 0.0000 \\
278 & 0.0001 & 0.0000 & 0.0000 \\
279 & 0.0000 & 0.0000 & 0.0000 \\
\hline
\end{tabular}

Therefore, we have

$$
\begin{aligned}
\left\|x_{k}-q\right\|^{2} \leq & \left\|x_{m_{k}+1}-q\right\|^{2} \leq \frac{\alpha_{m_{k}}\left\|x_{m_{k}}-q\right\|^{2}}{2(1-\rho)} \\
& +\frac{\left\langle f(q)-q, x_{m_{k}+1}-q\right\rangle}{1-\rho}+\frac{\left\|e_{m_{k}}\right\|}{\alpha_{m_{k}}} M_{1} \\
& +\frac{\left(1-\alpha_{m_{k}}-\beta_{m_{k}}\right)}{\alpha_{m_{k}}} M_{2},
\end{aligned}
$$

where

$$
\begin{aligned}
& M_{1}=\frac{\beta_{m_{k}}^{2}}{1-\rho}\left(\left\|z_{m_{k}}+e_{m_{k}}-q\right\|+2 \alpha_{m_{k}}\left\|x_{m_{k}+1}-q\right\|\right), \\
& M_{2}=\frac{1}{1-\rho}\left\|e_{m_{k}}-q\right\|\left\|x_{m_{k}+1}-q\right\| .
\end{aligned}
$$

From our assumptions $\lim _{k \longrightarrow \infty} \alpha_{m_{k}}=0, \lim _{k \longrightarrow \infty}(1-$ $\left.\alpha_{m_{k}}-\beta_{m_{k}}\right) / \alpha_{m_{k}}=0, \quad \lim _{k \rightarrow \infty}\left\|e_{m_{k}}\right\| / \alpha_{m_{k}}=0, \quad$ and (adjusted), we conclude $\lim \sup _{k \rightarrow \infty}\left\|x_{k}-q\right\|^{2}=0$. Hence, $x_{k} \longrightarrow q$ which completes the proof of part (ii).

Open problem 1. Can we remove the condition $\liminf _{n \longrightarrow \infty}\left\|x_{n}-y_{n}\right\|=0$ in (i) in Theorem 6?

\section{Numerical Example}

In this section, the algorithm (14) is illustrated with some examples.

Example 1. Put $\alpha_{n}=1 / n+1, \beta_{n}=1-1 / n+1, e_{n}=0, H=L_{2}$ $[0,1], A \equiv I, F(x) \equiv 1, \lambda=1 / 2, C=B(0,1)=\left\{f \in L_{2}[0,1]:\|f\|\right.$ $\leq 1\}$. 


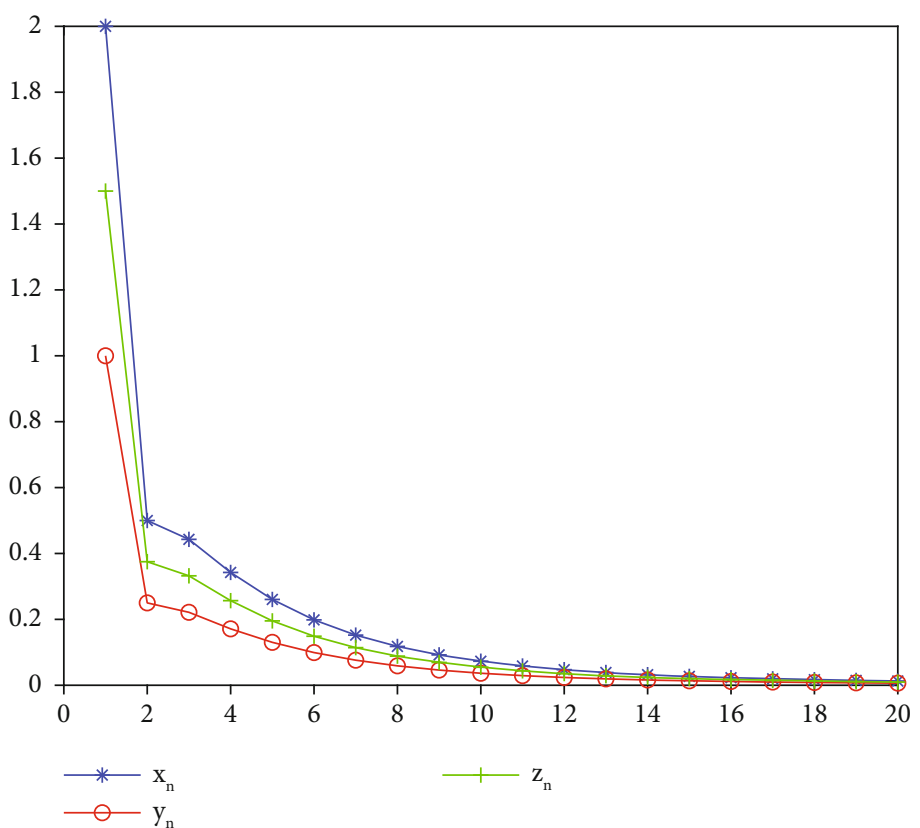

Figure 3: Convergence behavior $\left\{x_{n}\right\}$ in Example 3.

Then, from the algorithm (14), we have the following sequences:

$$
\begin{aligned}
g_{n} & =P_{C}\left(f_{n}-\lambda A f_{n}\right)=P_{C}\left(\frac{f_{n}}{2}\right), \\
k_{n} & =g_{n}-\lambda\left(A g_{n}-A f_{n}\right)=\frac{1}{2}\left(f_{n}+g_{n}\right), \\
f_{n+1} & =\alpha_{n} F\left(f_{n}\right)+\beta_{n} k_{n}+e_{n}=\frac{1}{n+1}+\frac{n}{n+1} k_{n},
\end{aligned}
$$

where $P_{C}$ is as follows

$$
P_{B(z, \rho)}(x)= \begin{cases}x & \|x-z\| \leq \rho, \\ z+\frac{\rho}{\|x-z\|}(x-z) & \|x-z\|>\rho,\end{cases}
$$

where $\rho>0$ (see [19]). We have,

$$
\begin{aligned}
\operatorname{VI}(C, A) & =\{f \in C:\langle A f, g-f\rangle \geq 0, \forall g \in C\} \\
& =\{f \in C:\langle f, g-f\rangle \geq 0, \forall g \in C\} .
\end{aligned}
$$

Obviously, $0 \in \operatorname{VI}(C, A)$; hence, $\operatorname{VI}(C, A) \neq \varnothing$.

Now, with $f_{1}=2$ and using the MATLAB software, we see that $\left\{f_{n}\right\}$ converges to 0 (Figure 1 ).

In the following example, using the MATLAB software, we compare some similar algorithms and their convergence speed and behavior. In particular, the TEGM algorithm (3), VSEGM algorithm (4), THEGM algorithm (6), and the algorithm (14) are compared. We see that the algorithm (14) has a higher convergence speed than the other algorithms (Figure 2).
Example 2. Let $\alpha_{n}=1 / \sqrt{n}, \beta_{n}=1-1 / \sqrt{n}-1 / n^{2}, \lambda=1 / 2, e_{n}$ $=0, H=\mathbb{R}, A \equiv I, f(x)=x / 2$, and $C=[0, \infty), x_{1}=2$ (Figure 2 ).

Now, we examine the convergence of the sequences $\left\{x_{n}\right\},\left\{y_{n}\right\}$, and $\left\{z_{n}\right\}$ in Theorem 6 in the following example.

Example 3. Put $\alpha_{n}=1 / \sqrt{n}, \beta_{n}=1-1 / \sqrt{n}-1 / n^{2}, \lambda=1 / 2, e_{n}$ $=1 / n^{2}, H=\mathbb{R}, A \equiv I, f \equiv 1$, and $C=[0, \infty), x_{1}=2$.

Then, we have

$$
\begin{aligned}
y_{n} & =P_{C}\left(x_{n}-\lambda A x_{n}\right)=P_{C}\left(\frac{1}{2} x_{n}\right), \\
z_{n} & =y_{n}-\lambda\left(A y_{n}-A x_{n}\right)=\frac{1}{2}\left(y_{n}+x_{n}\right), \\
x_{n+1} & =\alpha_{n} f\left(x_{n}\right)+\beta_{n} z_{n}+e_{n}=\frac{1}{\sqrt{n}}+\left(1-\frac{1}{\sqrt{n}}-\frac{1}{n^{2}}\right) z_{n}+\frac{1}{n^{2}} .
\end{aligned}
$$

Hence,

$$
\begin{aligned}
V I(C, A) & =\{x \in C:\langle A x, y-x\rangle \geq 0, \forall y \in C\} \\
& =\{x \in[0, \infty):\langle x, y-x\rangle \geq 0, \forall y \in C\}=\{0\} .
\end{aligned}
$$

Therefore, $q=P_{\mathrm{VI}(A, C)} \circ f(q)=P_{\{0\}} \circ(q / 2)=0$, and now, by Theorem 6 , the sequence $\left\{x_{n}\right\}$ converges strongly to 0 .

In the following example, we examine the case that $\operatorname{VI}(C, A)=\varnothing$, and the sequence generated by the algorithm (14) is divergent (Table 1 and Figure 3).

Example 4. Put $\alpha_{n}=1 / n, \beta_{n}=1-1 / n, \lambda=1 / 2, e_{n}=0, H=\mathbb{R}$, $A \equiv-1, f \equiv 1$, and $C=[0, \infty), x_{1}=2$. 


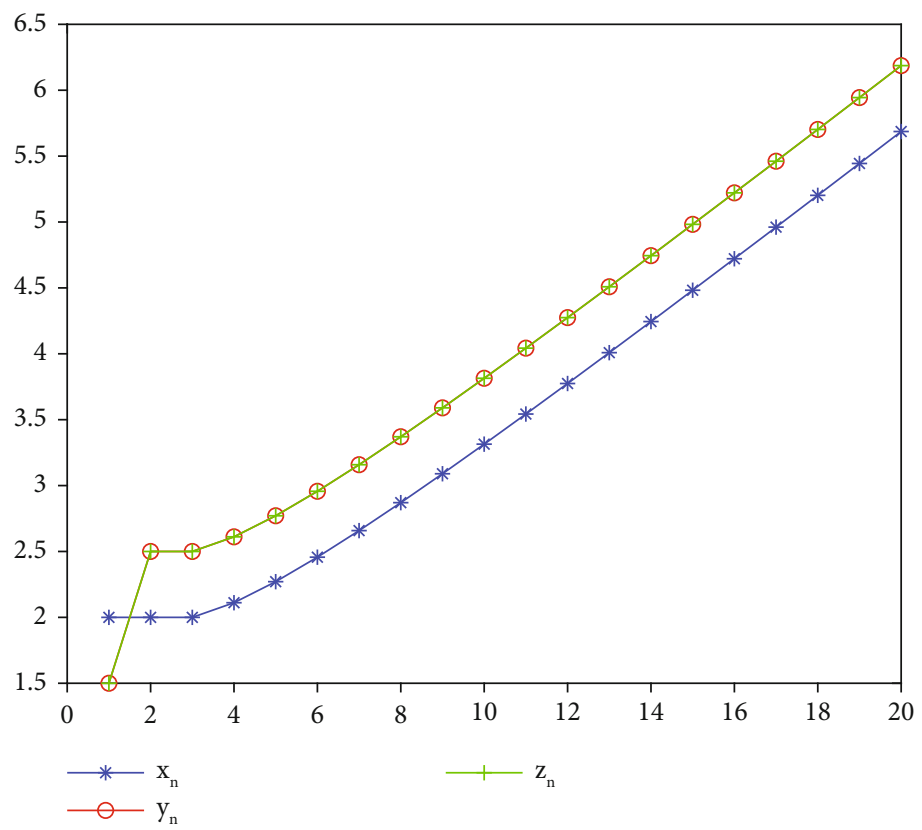

Figure 4: Convergence behavior $\left\{x_{n}\right\}$ in Example 4.

Then, we have

$$
\begin{aligned}
& y_{n}=P_{C}\left(x_{n}-\lambda A x_{n}\right)=P_{C}\left(x_{n}+\frac{1}{2}\right), \\
& z_{n}=y_{n}-\lambda\left(A y_{n}-A x_{n}\right)=y_{n}, \\
& x_{n+1}=\alpha_{n} f\left(x_{n}\right)+\beta_{n} z_{n}+e_{n}=\frac{1}{n}+\left(1-\frac{1}{n}\right) P_{C}\left(x_{n}+\frac{1}{2}\right) .
\end{aligned}
$$

Hence,

$$
\begin{aligned}
\operatorname{VI}(C, A) & =\{x \in C:\langle A x, y-x\rangle \geq 0, \forall y \in C\} \\
& =\{x \in[0, \infty):\langle-1, y-x\rangle \geq 0, \forall y \in C\} \\
& =\varnothing
\end{aligned}
$$

Now, we will prove by induction that, for all $n \in \mathbb{N}$

$$
y_{n}=P_{C}\left(x_{n}+\frac{1}{2}\right)=x_{n}+\frac{1}{2} .
$$

When $n=1$, then we have $x_{1}=2 \geq-1 / 2$.

Induction step: suppose for $n=k$ the inequality $x_{k} \geq-1 / 2$ holds.
Then,

$$
\begin{aligned}
x_{k+1} & =\frac{1}{k}+\left(1-\frac{1}{k}\right) P_{C}\left(x_{k}+\frac{1}{2}\right) \\
& =\frac{1}{k}+\left(1-\frac{1}{k}\right)\left(x_{k}+\frac{1}{2}\right) \\
& =\frac{1}{k}+\left(1-\frac{1}{k}\right) x_{k}+\left(1-\frac{1}{k}\right) \frac{1}{2} \\
& \geq \frac{1}{k}+\left(1-\frac{1}{k}\right)\left(-\frac{1}{2}\right)+\left(1-\frac{1}{k}\right) \frac{1}{2} \\
& \geq-\frac{1}{2} .
\end{aligned}
$$

Hence, $y_{n}=x_{n}+1 / 2$. Consequently $\lim _{n \rightarrow \infty}\left\|x_{n}-y_{n}\right\|=1 /$ $2 \neq 0$.

Next, we show that $\left\{x_{n}\right\}$ is an unbounded sequence. Note $x_{3}=5 / 4>3 / 4$. holds.

Induction step: suppose for $n=k$ the inequality $x_{k}>k / 4$

Then,

$$
\begin{aligned}
x_{k+1} & =\frac{1}{k}+\left(1-\frac{1}{k}\right)\left(x_{k}+\frac{1}{2}\right)>\frac{1}{k}+\left(1-\frac{1}{k}\right)\left(\frac{k}{4}+\frac{1}{2}\right) \\
& =\frac{1}{k}+\frac{k}{4}-\frac{1}{4}+\frac{1}{2}-\frac{1}{2 k}=\frac{1}{2 k}+\frac{k}{4}+\frac{1}{4}>\frac{k+1}{4},
\end{aligned}
$$

thus $\left\{x_{n}\right\}$ is an unbounded sequence (Figure 4). 


\section{Conclusions}

In this paper, we proposed a Tseng-type viscosity algorithm based on the viscosity method, which is an extension of the Thong et al.'s algorithm [3]. We showed that the sequence generated by the proposed algorithm strongly converges to an element of VI $(C, A)$.

The following are the results in this paper:

(i) We extended the results of Thong et al.'s. method [3] and provided necessary and sufficient conditions for the $\mathrm{VI}(C, A)$ to be nonempty

(ii) In our generated sequence $\left\{x_{n}\right\}$, in the algorithm (14), we put a sequence of computational errors $\left\{e_{n}\right\}$ and proved the convergence of the sequence in the presence of computational errors

(iii) We provided some numerical examples to compare our algorithm with the algorithms TEGM, VSEGM, and THEGM

\section{Data Availability}

No data were used to support the study.

\section{Conflicts of Interest}

This work does not have any conflicts of interest.

\section{References}

[1] R. P. Agarwal, D. O'Regan, and D. R. Sahu, Fixed Point Theory for Lipschitzian-Type Mappings with Applications, vol. 6, Springer, New York, 2009.

[2] R. Y. Apostol, A. A. Grynenko, and V. V. Semenov, "Iterative algorithms for monotone bilevel variational inequalities," The Journal of Computational and Applied Mathematics, vol. 107, pp. 3-14, 2012.

[3] D. V. Thong and D. V. Hieu, "Weak and strong convergence theorems for variational inequality problems," Numerical Algorithms, vol. 78, pp. 1045-1060, 2018.

[4] D. V. Thong, N. T. Vinh, and Y. J. Cho, "A strong convergence theorem for Tsengs extragradient method for solving variational inequality problems," Optimization Letters, vol. 14, no. 5, pp. 1157-1175, 2020.

[5] G. Bigi, M. Castellani, M. Pappalardo, and M. Passacantando, "Brcal protein products: functional motifsexistence and solution methods for equilibria," European Journal of Operational Research, vol. 227, pp. 1-11, 2013.

[6] D. B. Rouhani and S. Moradi, "Strong convergence of regularized new proximal point algorithms," Journal of Optimization Theory and Applications, vol. 181, pp. 864-882, 2019.

[7] D. B. Rouhani and S. Moradi, "Strong convergence of two proximal point algorithms with possible unbounded error sequences," Journal of Optimization Theory and Applications, vol. 172, pp. 222-235, 2017.

[8] A. Gibali, S. Reich, and R. Zalas, "Outer approximation methods for solving variational inequalities in hilbert space," Optimization, vol. 66, pp. 417-437, 2017.
[9] C. Kanzow and Y. Shehu, "Generalized Krasnoselskii-Manntype iterations for nonexpansive mappings in Hilbert spaces," Computational Optimization and Applications, vol. 67, no. 3, pp. 595-620, 2017.

[10] R. Kraikaew and S. Saejung, "Strong convergence of the Halpern subgradient extragradient method for solving variational inequalities in Hilbert spaces," Journal of Optimization Theory and Applications, vol. 163, pp. 399-412, 2014.

[11] Y. V. Malitsky and V. V. Semenov, "A hybrid method without extrapolation step for solving variational inequality problems," Journal of Global Optimization, vol. 61, pp. 193-202, 2015.

[12] Y. M. Wang, Y. B. Xiao, X. Wang, and Y. J. Cho, "Equivalence of well-posedness between systems of hemivariational inequalities and inclusion problems," Journal of Nonlinear Sciences and Applications, vol. 9, pp. 1178-1192, 2016.

[13] G. M. Korpelevich, "The extragradient method for finding saddle points and other problems," Ekonomikai Matematicheskie Metody, vol. 12, pp. 747-756, 1976.

[14] P. Tseng, "A modified forward-backward splitting method for maximal monotone mappings," SIAM Journal on Control and Optimization, vol. 38, pp. 341-446, 2000.

[15] Y. Shehu and O. S. Iyiola, "Strong convergence result for monotone variational inequalities," Numerical Algorithms, vol. 76, no. 1, pp. 259-282, 2017.

[16] H. K. Xu, "Iterative algorithms for nonlinear operators," Journal of the London Mathematical Society, vol. 66, pp. 240-256, 2002.

[17] K. Goebel and S. Reich, Uniform Convexity, Hyperbolic Geometry, and Nonexpansive Mappings, Marcel Dekker, New York, 1984.

[18] F. Mainge, "A hybrid extragradient-viscosity method for monotone operators and fixed point problems," SIAM Journal on Control and Optimization, vol. 47, pp. 1499-1515, 2008.

[19] A. Cegielski, "Iterative methods for fixed point problems in hilbert spaces," Nature Genetics, vol. 13, pp. 266-267, 1996. 